\title{
Hydrogen Interactions With Semiconductors And Oxides
}

\author{
Chris G. Van de Walle \\ Palo Alto Research Center, Palo Alto, CA 94304
}

\begin{abstract}
Hydrogen plays an important role as an impurity in solids. Hydrogen's interactions with materials are discussed on the basis of its behavior as an isolated interstitial impurity. In most semiconductors and oxides hydrogen is amphoteric, always counteracting the prevailing conductivity of the material. But in some materials hydrogen acts as a source of conductivity. These concepts are illustrated with the example of hydrogen in zinc oxide.
\end{abstract}

\section{INTRODUCTION}

Hydrogen displays a range of complex behaviors when introduced as an impurity in solids. Interstitial hydrogen is a fast diffuser. It can bind to native defects or to other impurities, often eliminating their electrical activity - a phenomenon known as passivation. But hydrogen can also induce electrically active defects. Various technological developments are lending new urgency to a fuller understanding of the behavior of hydrogen in solids: the search for improved hydrogen storage systems and proton exchange membranes for fuel cells; the pursuit of novel dielectrics for integrated circuits (in which hydrogen may play an equally important role as it does at the $\mathrm{Si} / \mathrm{SiO}_{2}$ interface); and efforts to take advantage of the passivation of dopant impurities during growth to enhance doping levels (codoping).

Over the past 20 years we have learnt a great deal about hydrogen in semiconductors, based on experiment as well as theory. Many aspects of hydrogen's interactions with materials can be understood on the basis of the behavior of hydrogen as an isolated interstitial impurity. In most semiconductors hydrogen is amphoteric: $\mathrm{H}$ acts as a donor $\left(\mathrm{H}^{+}\right)$in $p$-type material, and as an acceptor $\left(\mathrm{H}^{-}\right)$in $n$-type material, always counteracting the prevailing conductivity. For instance, in $\mathrm{GaN}$ hydrogen passivates $\mathrm{Mg}$ acceptors, and needs to be removed in a post-growth anneal in order to activate the acceptors [1]. Subsequent experiments and first-principles calculations for a number of other semiconductors seemed to confirm this behavior as a general feature of hydrogen's interactions with semiconductors. It therefore came as a surprise when calculations showed that hydrogen behaves exclusively as a donor in $\mathrm{ZnO}$ [2]. Only the positive charge state is stable in $\mathrm{ZnO}$, and therefore hydrogen can act as a source of doping, rather than merely reducing the conductivity introduced by other dopants. The donor character of hydrogen in $\mathrm{ZnO}$ obviously has a number of practical consequences. However, maybe the most interesting issue is: what is the fundamental reason for hydrogen to behave differently in $\mathrm{ZnO}$ ? In the course of investigating this

CP671, Hydrogen in Materials and Vacuum Systems edited by G. R. Myneni and S. Chattopadhyay

(C) 2003 American Institute of Physics 0-7354-0137-3/03/\$20.00 
question we have been able to predict that hydrogen will act as a source of doping in a number of other materials as well, including InN [3] and InGaAsN alloys [4], where first-principles calculations have already confirmed the behavior. A general model for predicting the behavior of $\mathrm{H}$ in semiconductors and oxides is discussed.

I will also discuss a specific example of hydrogen passivation of point defects. In $\mathrm{ZnO}$, hydrogen in an oxygen vacancy acts as a shallow donor, while hydrogen in a zinc vacancy passivates the defect, and suppresses the green luminescence associated with zinc vacancies [5].

\section{METHODS}

We use first-principles calculations to study the behavior of hydrogen in a wide range of materials. The approach is based on density-functional theory (DFT) within the local density approximation (LDA) and the pseudopotential-plane-wave method. The key quantities that determine the properties of hydrogen are the formation energy, i.e., the energy needed to incorporate $\mathrm{H}$ in the host, and the electronic transition level, which defines the electrical behavior. The transition level $\varepsilon(+/-)$ between the positive and negative charge states is defined as the Fermi-level position for which the formation energies of these charge states are equal. The calculations for interstitial $\mathrm{H}$ were carried out in supercells containing either 64 atoms for zinc-blende or 96 atoms for wurtzite. For $\mathrm{H}$ the Coulomb potential is used. Energy differences for hydrogencontaining systems are well converged at a 40 Ry plane-wave cutoff. For each charge state of $\mathrm{H}$, many possible sites in the lattice were explored and the global minimum was identified. Further details can be found in Ref. [1].

\section{INTERSTITIAL HYDROGEN}

\section{Hydrogen in GaN}

Figure 1 illustrates the basic concepts with the example of hydrogen in $\mathrm{GaN} . \mathrm{H}^{0}$ is never the lowest-energy state (characteristic of a "negative- $U$ " system), causing the donor level $\varepsilon(+/ 0)$ to lie above the acceptor level $\varepsilon(0 /-)$. When $E_{F}$ moves through the band gap, the stable charge state thus changes directly from positive (for $E_{F}$ below 2.4 $\mathrm{eV}$ ) to negative (for $E_{F}$ above $2.4 \mathrm{eV}$ ). This implies that in $p$-type $\mathrm{GaN}$ [ $E_{F}$ close to the valence-band maximum $(\mathrm{VBM})] \mathrm{H}^{+}$is favored, while in $n$-type $\mathrm{GaN}$ [ $E_{F}$ close to the conduction-band minimum $(\mathrm{CBM})] \mathrm{H}^{-}$is stable, providing the basis for hydrogen's tendency to counteract the prevailing conductivity. The Fermi-level position where the positive and negative charge states are equal in energy is labeled $\varepsilon(+/-)$, and plays a crucial role in the theory. This behavior of hydrogen in $\mathrm{GaN}$ is qualitatively very similar to what has been observed or calculated for other materials $[1,6]$, although quantitatively the values of formation energies and transition levels vary over a large range. 


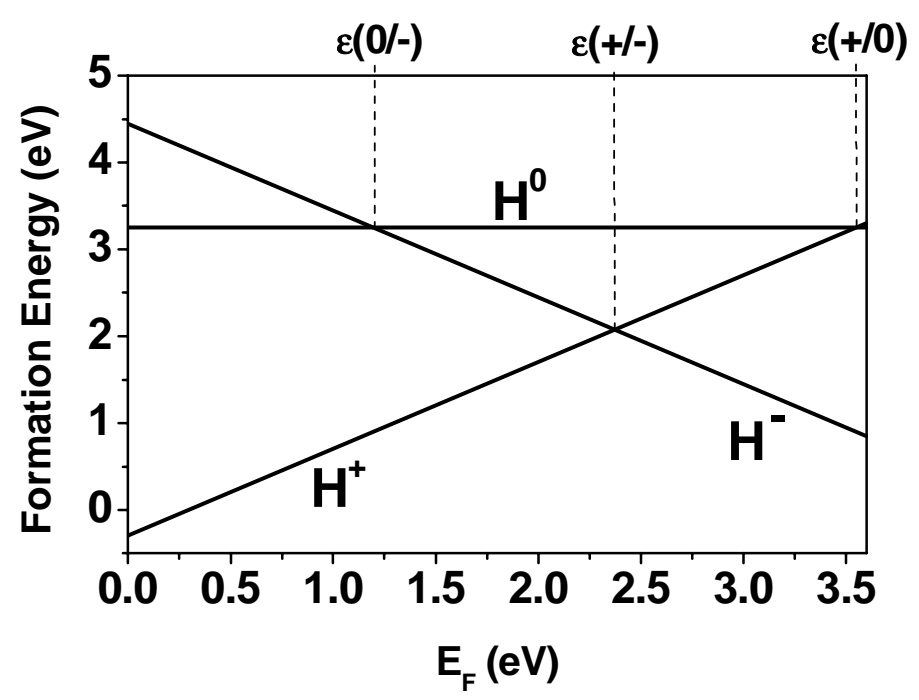

FIGURE 1. Formation energy of interstitial hydrogen in wurtzite GaN as a function of Fermi level, obtained from DFT-LDA calculations. $\mathrm{E}_{\mathrm{F}}=0$ corresponds to the valence-band maximum, and formation energies are referenced to the energy of an $\mathrm{H}_{2}$ molecule.

\section{Hydrogen in $\mathrm{ZnO}$}

In some materials, however, hydrogen exhibits a completely different behavior: for instance, in $\mathrm{ZnO} \mathrm{H}^{0}$ and $\mathrm{H}^{-}$are always higher in energy than $\mathrm{H}^{+}$, for any Fermi-level position [2]. The fact that only $\mathrm{H}^{+}$is stable implies that hydrogen acts as a shallow donor, i.e., as a source of conductivity. In this case, $\varepsilon(+/-)$ does not lie in the band gap, but above the conduction-band minimum (CBM).

$\mathrm{H}^{+}$in $\mathrm{ZnO}$ prefers sites where it can strongly bind to an oxygen atom, forming an $\mathrm{O}-\mathrm{H}$ bond with a length of 0.99-1.01 $\AA$. Large lattice relaxations occur around the hydrogen interstitial; in particular, for the bond-center (BC) configuration the $\mathrm{Zn}$ atom moves outward over a distance equal to $40 \%$ of the bond length $(0.8 \AA)$, to a position slightly beyond the plane of its nearest neighbors. Simultaneously, the $\mathrm{O}$ atom moves outwards by $11 \%$ of the bond length.

We have also studied substitutional hydrogen on an oxygen site (which can also be regarded as a complex consisting of an oxygen vacancy and a hydrogen atom). The calculated binding energy, expressed with respect to $\mathrm{H}^{+}$and $V_{\mathrm{O}}$, is $0.8 \mathrm{eV}$. The hydrogen atom is located close to the nominal oxygen lattice position (to within $0.05 \AA$ ). We have found that substitutional hydrogen also behaves as a shallow donor.

The fact that only the positive charge state of hydrogen is stable in $\mathrm{ZnO}$ implies that $\mathrm{H}$ can act as a dopant in this material. Indeed, unlike the situation in $\mathrm{GaN}$, hydrogen in $\mathrm{ZnO}$ does not self-compensate. No matter how close we drive the Fermi level to the conduction band, hydrogen continues to act as a donor. This result has important consequences for $\mathrm{ZnO}$ technology. 
$\mathrm{ZnO}$ indeed has numerous technological applications, including in varistors, transducers, phosphors, and sensors. A recent resurgence of interest in $\mathrm{ZnO}$ has occurred due to advances in bulk crystal as well as thin-film growth, and because of potential new applications of $\mathrm{ZnO}$ in light-emitting devices. $\mathrm{ZnO}$ has a band gap of 3.4 $\mathrm{eV}$, very close to that of $\mathrm{GaN}$; one may thus envision using it for similar applications as $\mathrm{GaN}$ in optoelectronics, i.e., light emitting diodes and lasers in the blue and UV region of the spectrum. Efficient excitonic emission should be possible at room temperature due to the large exciton binding energy $(60 \mathrm{meV})$. For comparison, the exciton binding energy in $\mathrm{GaN}$ is $25 \mathrm{meV}$. Optically pumped lasing has already been reported in $\mathrm{ZnO}$ platelets [7], thin films [8], clusters consisting of $\mathrm{ZnO}$ nanocrystals [9], and $\mathrm{ZnO}$ nanowires [10].

$\mathrm{ZnO}$ can be grown with a variety of techniques, and most of these produce $\mathrm{ZnO}$ that exhibits residual $n$-type conductivity, sometimes at a very high level. This high level of $n$-type conductivity may be useful for some applications, such as transparent conductors that can be used for solar cells or displays, but in general it would be desirable to have better control over the conductivity. In particular, the ability to reduce the $n$ type background and to achieve $p$-type doping would open up tremendous possibilities for device applications in general and for light-emitting diodes and lasers in particular. A fundamental understanding of which impurities can act as donors in $\mathrm{ZnO}$ is therefore very important. In particular, since hydrogen is an omnipresent impurity, and since many of the growth techniques either intentionally or unintentionally introduce hydrogen, it should come as no surprise that hydrogen would play a role in the unintentional $n$-type doping.

Experimental indications for hydrogen's behavior as a donor in $\mathrm{ZnO}$ were already reported in the 1950s [11,12], and the nature of hydrogen as a donor impurity has conclusively emerged from recent experiments. Muon spin rotation is a technique similar to electron paramagnetic resonance (EPR), based on muonium, which is a pseudoisotope of hydrogen. Muonium in $\mathrm{ZnO}$ was observed to exhibit all characteristics of a shallow donor, including ionization behavior consistent with a level close to the conduction band, and a delocalized wavefunction [13,14]. Electron paramagnetic resonance has also recently resulted in a direct observation of hydrogen in $\mathrm{ZnO}$, with behavior consistent with a shallow donor [15]. Hydrogen was identified as one of two residual donors in commercial $\mathrm{ZnO}$ samples; the presence of two donors in this material is consistent with Hall measurements. The involvement of hydrogen in the structure of one of these donors was confirmed by electron-nuclear double resonance (ENDOR).

It is an interesting question whether these experiments observe the interstitial or the substitutional species. Because of the way the muons are introduced in the $\mathrm{ZnO}$ material, we believe the muon spin rotation experiments observe the equivalent of isolated interstitial hydrogen. In the EPR experiments on commercial $\mathrm{ZnO}$ however, we suspect the substitutional species may be observed, based on its greater stability. 


\section{General theory of hydrogen in semiconductors}

As discussed above, hydrogen in $\mathrm{ZnO}$ behaves very differently from hydrogen in the semiconductors that were previously studied, including $\mathrm{Si}$, GaAs, GaN, etc. In the latter, hydrogen is an amphoteric impurity, corresponding to the notion that the $\varepsilon(+/-)$ transition level occurs well within the band gap (as we saw in the case of GaN). In $\mathrm{ZnO}$, however, only $\mathrm{H}^{+}$is stable, meaning that $\varepsilon(+/-)$ occurs above the conductionband minimum (CBM). The crucial question is why $\varepsilon(+/-)$ for $\mathrm{H}$ in $\mathrm{ZnO}$ occurs in such a different energetic position (above the CBM) compared to, say, $\varepsilon(+/-)$ in $\mathrm{GaN}$ (at $2.4 \mathrm{eV}$ above the valence band). A detailed analysis showed no correlation of the position of the charge transfer level with ionicity, band gap, cohesive energy, or hydrogen formation energy. However, we have discovered a direct link with the position of the band structure on an absolute energy scale [16]. Indeed, despite the similarity of their band gaps, the band offsets between $\mathrm{GaN}$ and $\mathrm{ZnO}$ are quite large. The calculated valence-band offset is about $1.3 \mathrm{eV}$. Assuming that the hydrogen $\varepsilon(+/-)$ level occurs at the same "absolute" energy across various semiconductors, its position at $E_{\mathrm{v}}+2.4 \mathrm{eV}$ in $\mathrm{GaN}$ would place it at $E_{\mathrm{v}}+2.4+1.3=E_{\mathrm{v}}+3.7 \mathrm{eV}$ in $\mathrm{ZnO}$, i.e., well above the $\mathrm{ZnO} \mathrm{CBM}$ - consistent with our explicit first-principles results for hydrogen in $\mathrm{ZnO}$.

Encouraged by this agreement, we have investigated the assumption that the $\varepsilon(+/-)$ would be constant on an absolute energy scale for a wider range of materials. A combination of first-principles calculations for hydrogen levels and for band lineups has indeed confirmed that this is the case [16]. The correlation has in fact already allowed several interesting predictions. For instance, the expected value of $\varepsilon(+/-)$ in InN lies well above the CBM, a prediction that was confirmed by explicit first-principles calculations [3] that indeed showed that only $\mathrm{H}^{+}$is stable in InN. Subsequently, muon-spin rotation has confirmed the shallow-donor character [17], and experimental investigations of InN grown with gas-source molecular beam epitaxy [18] have also shown that the electron concentrations in InN exceed the concentrations of known shallow donors such as oxygen or silicon, suggesting that hydrogen (which is present in high concentrations) is causing the $n$-type conductivity.

Another case that was recently studied is GaAsN. Although $\varepsilon(+/-)$ lies well within the band gap for both GaAs and $\mathrm{GaN}$, we speculated that the large band-gap bowing observed in GaAsN alloys could push the CBM far enough down to cause $\varepsilon(+/-)$ to lie above it. Again, explicit calculations [4] have now confirmed this prediction. The shallow donor nature of hydrogen is even stronger in the technologically important InGaAsN alloys, which have an even lower CBM than GaAsN.

\section{HYDROGEN INTERACTIONS WITH POINT DEFECTS}

It is well known that hydrogen can passivate point defects. An interesting example of such interactions has recently been studied, both experimentally and theoretically, in $\mathrm{ZnO}$ [5]. We mentioned above that hydrogen in an oxygen vacancy acts as a shal- 
low donor, i.e., it is an electrically active defect. But hydrogen has a distinct passivating effect on the zinc vacancy. We have previously proposed that the zinc vacancy in $\mathrm{ZnO}$ is a possible source of the frequently observed green luminescence [19]. Sekiguchi et al. [20] have observed that a hydrogen plasma treatment strongly reduces the intensity of the green luminescence. We have therefore investigated the interaction between hydrogen and zinc vacancies. The $\mathrm{Zn}$ vacancy is a double acceptor and occurs in the 2- charge state $\left(V_{\mathrm{Zn}}{ }^{2-}\right)$ in $n$-type $\mathrm{ZnO}$. This immediately suggests that the vacancy can be neutralized by binding two hydrogen atoms. We find, indeed, that the binding energy per $\mathrm{H}$, referenced to interstitial $\mathrm{H}^{+}$, is $1.80 \mathrm{eV}$. Passivation of $V_{\mathrm{Zn}}$ with two hydrogen atoms eliminates the optical activity of the center. One of the hydrogen atoms forms an $\mathrm{O}-\mathrm{H}$ bond oriented approximately along the $c$ axis (our calculations actually produce an angle with the $c$ axis of $10^{\circ}$ ). The other hydrogen forms an $\mathrm{O}-\mathrm{H}$ bond at an angle of $98^{\circ}$ with the $c$ axis; i.e., it is tilted away from the nominal $\mathrm{Zn}-\mathrm{O}$ bond direction in the perfect crystal by about $11^{\circ}$, towards a direction perpendicular to the $c$ axis. We have calculated the vibrational frequencies of the stretch modes corresponding to the $\mathrm{O}-\mathrm{H}$ bonds. These vibrational frequencies have been found to be in good agreement with the values obtained from infrared absorption spectroscopy on hydrogenated samples [5].

\section{CONCLUSIONS}

Hydrogen strongly affects the electronic properties of materials. We have given examples of cases where hydrogen passivates the electrical and optical activity of other centers, but hydrogen can also serve as a source of doping in its own right. A general model for predicting the electrical behavior of hydrogen in semiconductors and oxides has been developed.

\section{ACKNOWLEDGMENTS}

This work was supported in part by AFOSR under contract number F49620-02-1-1163. I gratefully acknowledge collaborations and discussions with J. Neugebauer S. Limpijumnong, M. Fuchs, M. Chabinyc, D. Biegelsen, E. Lavrov, J. Weber, and the late J. McCaldin.

\section{REFERENCES}

1. Neugebauer, J., and Van de Walle, C. G., "Theory of hydrogen in GaN," in Hydrogen in Semiconductors II, Semiconductors and Semimetals Vol. 61, edited by N. H. Nickel, Boston:Academic Press, Boston, 1999, pp. 479-502.

2. Van de Walle, C. G., Phys. Rev. Lett. 85, 1012-1015 (2000).

3. Limpijumnong, S., and Van de Walle, C. G., phys. stat. sol. (b) 228, 303-307 (2001).

4. Janotti, A., Zhang, S., Wei, S., and Van de Walle, C. G., Phys. Rev. Lett. 89, 086403 (2002). 
5. Lavrov, E. V., Weber, J., Börrnert, F., Van de Walle, C. G., and Helbig R., Phys. Rev. B 66, 165205 (2002).

6. Van de Walle, C. G., and Johnson, N. M., "Hydrogen in III-V Nitrides," in Gallium Nitride (GaN) II, Semiconductors and Semimetals Vol. 57, edited by J. I. Pankove and T. D. Moustakas, Boston:Academic Press, 1998, pp. 157-184.

7. Reynolds, D. C., Look, D. C., and Jogai, B., Solid State Commun. 99, 873-875 (1996).

8. Bagnall, D. M., Chen, Y. F., Zhu, Z., Yao, T., Koyama, S., Shen, M. Y., and Goto, T., Appl. Phys. Lett. 70, 2230-2232 (1997).

9. Cao, H., Xu, J. Y., Seelig, E. W., and Chang, R. P. H., Appl. Phys. Lett. 76, 2997-2999 (2000).

10. Huang, M. H., Mao, S., Feick, H., Yan, H. Q., Wu, Y. Y., Kind, H., Weber, E., Russo, R., and Yang, P. D., Science 292, 1897-1899 (2001).

11. Mollwo, E., Z. Physik 138, 478-488 (1954).

12. Thomas, D. G., and Lander, J. J., J. Chem. Phys. 25, 1136-1142 (1956).

13. Cox, S. F. J., Davis, E. A., Cottrell, S. P., King, P. J. C., Lord, J. S., Gil, J. M., Alberto, H. V., Vilão, R. C., Piroto Duarte, J., Ayres de Campos, N., Weidinger, A., Lichti, R. L., and Irvine, S. J. C., Phys. Rev. Lett. 86, 2601-2604 (2001).

14. Shimomura, K., Nishiyama, K., and Kadono, R., Phys. Rev. Lett. 89, 255505 (2002).

15. Hofmann, D. M., Hofstaetter, A., Leiter, F., Zhou, H., Henecker, F., Meyer, B. K., Orlinskii, S. B., Schmidt, J., and Baranov, P. G., Phys. Rev. Lett. 88, 045504 (2002).

16. Van de Walle, C. G., and Neugebauer, J. (unpublished).

17. Davis, E. A., Cox, S. F. J., Lichti, R. L., and Van de Walle, C. G., Appl. Phys. Lett. 82, 592 (2003).

18. Look, D. C., Lu, H., Schaff, W. J., Jasinski, J., and Lilienthal-Weber, Z., Appl. Phys. Lett. 80, 258 (2002).

19. Kohan, A. F., Ceder, G., Morgan, D., and Van de Walle, C. G., Phys. Rev. B 61, 15019-27 (2000).

20. Sekiguchi, T., Ohashi, N., and Terada, Y., Jpn. J. Appl. Phys. 36, L289-91 (1997). 